\title{
Influence of essential amino acids on muscle mass and muscle strength in patients with cerebral stroke during early rehabilitation: protocol and rationale of a randomized clinical trial (AMINO-Stroke Study)
}

Nadja Scherbakov ${ }^{1,2}$ (D) Nicole Ebner ${ }^{3}$, Anja Sandek ${ }^{3}$, Andreas Meisel ${ }^{1,4}$, Karl Georg Haeusler ${ }^{1,4}$, Stephan von Haehling ${ }^{3}$, Stefan D. Anker ${ }^{3}$, Ulrich Dirnagl 1,2,4 ${ }^{\text {Michael Joebges }}{ }^{5}$ and Wolfram Doehner ${ }^{1,2,6^{*}}$

\begin{abstract}
Background: Patients with stroke are at a high risk for long-term handicap and disability. In the first weeks after stroke muscle wasting is observed frequently. Early post-stroke rehabilitation programs are directed to improve functional independence and physical performance. Supplementation with essential amino acids (EAAs) might prevent muscle wasting and improve rehabilitation outcome by augmenting muscle mass and muscle strength. We aim to examine this in a double blinded, randomized placebo-controlled clinical trial.

Methods: Patients with ischemic or haemorrhagic stroke will be enrolled at begin of the early post-stroke rehabilitation in a parallel group interventional trial. Oral supplementation of EAAs or placebo will be given for 12 weeks in a double blinded manner. Physical and functional performance will be assessed by exercise testing before supplementation of EAAs as well as at discharge from the in-patient rehabilitation, at 12 weeks and 1 year afterwards.
\end{abstract}

Discussion: This is the first randomized double-blinded placebo-controlled clinical study aiming to assess the effect of the EAAs supplementation on muscle strength, muscle function and physical performance in stroke patients during early post-stroke rehabilitation. Supplementation of EAAs could prevent muscle mass wasting and improve functional independence after stroke.

Trial registration: The study is registered at the German registry for clinical trials as well as at World Health Organization (WHO; number DRKS00005577).

Keywords: Double blinded randomized study, Post-stroke rehabilitation, Skeletal muscle wasting, Physical performance, Essential amino acids

\footnotetext{
*Correspondence: wolfram.doehner@charite.de

${ }^{1}$ Center for Stroke Research Berlin CSB, Charité - Universitätsmedizin Berlin,

Berlin, Germany

${ }^{2}$ German Centre for Cardiovascular Research (DZHK), partner site Berlin,

Berlin, Germany

Full list of author information is available at the end of the article
} 


\section{Background}

Long-term disability and functional dependency are the main complications after stroke. Impaired skeletal muscle innervation due to damage results in the degeneration of motor units, paresis, and immobility accompanied by skeletal muscle atrophy [1-4]. Notably, loss of muscle mass and muscle function, defined as sarcopenia, have been originally described as a phenomenon of aging [5]. However, muscle wasting observed in stroke patients is a disease-related phenomenon and the term 'stroke-related sarcopenia' has been suggested [1]. The aetiology of sarcopenia is multifactorial [6]. This is also true for stroke-depended sarcopenia. Several pathophysiological mechanisms including metabolic imbalance, inactivity, malnutrition, and inflammation may contribute to the reduction of muscle mass after stroke $[7,8]$. Stroke-related muscle wasting is accompanied by body weight loss, neuro-hormonal activation, and a systemic shift towards catabolic over-activation [8]. In addition, activation of catabolic pathways in the skeletal muscle of the paretic and non-paretic limbs has been observed in experimental stroke [9].

Early rehabilitation has a great impact on the functional recovery after stroke. A previous study showed that $80 \%$ of the patients achieve best functional recovery within 6 weeks after stroke, and after 12.5 weeks $95 \%$ of all stroke patients completed their functional recovery [10]. However, one year after stroke more than $30 \%$ of the patients remained functionally dependent [11].

Skeletal muscles play a central role in post-stroke rehabilitation. The goal of rehabilitation is to restore functional muscle capacity and to prevent long-term disability. Therefore, prevention of muscle wasting, increasing muscle strength, reactive development of novel neuromuscular junctions, and stabilisation of the catabolic-anabolic imbalance are primary aims of poststroke rehabilitation.

Nutritional intervention may contribute to the improvement of muscle bulk and functional capacity in the early post-stroke rehabilitation. Thus, a beneficial effect of dietary supplementation of essential amino acids (EAAs) has been shown for an improvement of skeletal muscle mass and function in the elderly [12, 13]. Availability of EAAs is regarded as a limiting step in the synthesis of new muscle proteins [14]. Previously it has been shown that supplementation of EAAs in the elderly was responsible for amino acidinduced stimulation of muscle protein anabolism [15]. Therefore, an individually adjusted rehabilitation program that includes physical, functional and neuropsychological training as well as dietary supplementation of the EAA might provide the best rehabilitation outcome. Thus, in the present clinical trial the following hypotheses will be tested:
- Oral uptake of EAAs restores skeletal muscle function and physical performance;

- Oral uptake of EAAs contributes to physical independence and enhances the effectiveness of post-stroke rehabilitation;

- Availability of EAAs reduces muscle wasting after stroke.

We aim to demonstrate that nutritional supplementation with biologically limited available EAAs (l-leucine, l-lysine, l-isoleucine, l-valine, l-threonine, l-cystine, lhistidin, l-phenylalanine, l-methionine, l-tyrosin, and 1tryptophane), in synergy with individually adjusted physical training prevent muscle wasting after stroke and improve the effect of post-stroke early rehabilitation.

\section{Methods}

\section{Study design}

The "Influence of essential amino acids on muscle mass and muscle strength in patients with cerebral stroke during early rehabilitation (AMINO-Stroke)" study is a randomized double-blinded placebo controlled interventional clinical trial. The study is planed as a monocentric trial within the interdisciplinary trial programme of the Center for Stroke Research Berlin (CSB) and is funded by the German Federal Ministry of Education and Research (BMBF; grant number CSB 01EO1301). The study has been approved by the Brandenburg Ethics Committee for the recruiting site (S13(a)/2013) and is conducted in accordance with the Declaration of Helsinki. The study is registered at the German registry for clinical trials as well as at World Health Organization (WHO; number DRKS00005577).

\section{Patient eligibility}

Patients admitted to the rehabilitation centre Brandenburg Klinik, Bernau, Germany within 8 weeks after ischemic or haemorrhagic stroke will be screened for the eligibility. Patients of both genders, aged 45 years or older will be informed about the study. After obtaining of written informed consent from the patients they will be randomized 1:1 to intervention and control group by a web-based online randomization procedure provided by the Pharmacy at the Charité. For inclusion and exclusion criteria see Table 1.

\section{Planned clinical follow-up visits}

In order to evaluate an effect of the EAAs on muscle function, baseline functional assessment program will be performed at admission, followed by follow-up assessment at discharge from the in-patient rehabilitation (Table 2). All patients included into the study will receive 12 weeks either the EAAs ( 4 gram, 3 times a day) 
Table 1 Inclusion and exclusion criteria of the AMINO-Stroke

Inclusion criteria:

- Patient $>45$ years

- Patients with ischemic or haemorrhagic stroke within 8 weeks to enrolment

- Brain magnet resonance imaging or computer tomography demonstrating stroke

- Motoric disability of an upper and /or lower limb (Rivermead Motor Assessment Gross Function $>1$ and $<11$ )

- Signed informed consent

Exclusion criteria:

- Clinically significant findings on physical examination or presence of clinically significant disease that would interfere with study evaluation in the opinion of the treating physicians

- Participation in another clinical trial investigating a nutritional product

- History of intolerance or allergic response to similar nutritional products or known hypersensitivity to essential amino acids

- Clinical sighs and symptoms of infection requiring antibiotic therapy at the time of enrolment that prevent completion of trial-related assessments as judged by the investigator

- Transaminases (AST or ALT) $>3$ times the upper limit of normal (ULN)

- Severe renal dysfunction or nephrotic syndrome

- Acquired immunodeficiency syndrome, HIV or Hepatitis C infection

- Current therapy with anabolic steroids or appetite stimulants

- Current immunosuppressive therapy, heart transplantation, or renal dialysis

- Life expectancy $<6$ months

Table 2 Functional assessment at baseline and during follow-up

Extended functional assessment at baseline and at 4 weeks follow-up

- Activity of daily living (modified Rankin scale, Barthel Index)

- Functional assessment scales and physical performance (Rivermead Motor Assessment Gross Function, Fugl-Meyer Scale, Functional ambulatory capacity)

- Muscle functional assessment (SPPBT, hand grip, pinch grip)

- Quality of life and nutritional status (EQ-5D, PGA)

- Blood and biomarker bank: metabolic /immunologic profile

- $24 \mathrm{~h}$ Holter electrocardiogram (ECG)

- Body composition by bioelectrical impendance analysis (BIA)

- Urine status

Functional assessment at 3 and 6 months follow-up

- Activity of daily living (mRS, Barthel index)

- Muscle functional assessment (SPPBT, hand grip, pinch grip)

- Quality of life and nutritional status (EQ-5D, PGA)

- Blood and biomarker bank: metabolic/immunologic profile

- 24 h Holter ECG

- Body composition by bioelectrical impedance analysis (BIA)

- Urine status or placebo dissolved in a glass of water as a drinking solution.

Two weeks after the enrolment a study nurse visit for evaluation of compliance of the EEAs supplementation and tolerance to the compound is planned. After discharge from the in-patient rehabilitation, two follow-up visits at 3 and at 12 months are planned. Patients will be tested for functional performance and physical independence during a 1-day ambulatory visit. Patients, who are unable to visit the study centre will be contacted by telephone and interviewed using a standardised questionnaire. The study design is summarized in Fig. 1.

\section{Outcomes and endpoints \\ Primary outcomes}

Primary outcomes will be assessed at the 4 week followup visit. Primary endpoints include:

1) Physical performance according to the Rivermead motor assessment (RMA) gross function scale;

2) Muscle strength in a maximum hand grip strength test.

\section{Secondary outcomes}

Secondary endpoints include:

1) Functional outcome assessed by Barthel Index (BI);

2) Quality of life assessed according to EuroQoL (EQ)5D (EQ-5D) questionnaire at 3 and 12 months follow up visits;

3) Muscle function and motor capabilities assessed by pinch grip strength test, motor assessment scale, Fugl-Meyer Score (motor functional domain), Functional ambulatory capacity and short physical performance battery test;

4) Changes of body composition assessed by bioelectrical impedance analysis (BIA);

5) Changes in insulin sensitivity;

6) Changes of nutritional status according to Mini Nutritional Assessment questionnaire and metabolic profile according to biochemical analyses;

7) Rate of post-stroke complications, stroke -associated infections and re-hospitalization for any reason;

8) Safety and tolerability of EEAs supplementation.

\section{Physical examinations}

Rivermead motor assessment (RMA) gross function scale comprised a series of 13 questions, and covers a range of activities from turning over in bed to hop on affected leg five times [16, 17]. Hand grip strength [18] and pinch grip tests measures muscle isometric strength. The maximum value of three measurements will be evaluated [19]. Motor assessment scale (MAS) includes nine items scoring from 0 to 6 , and is designed to assess eight areas 


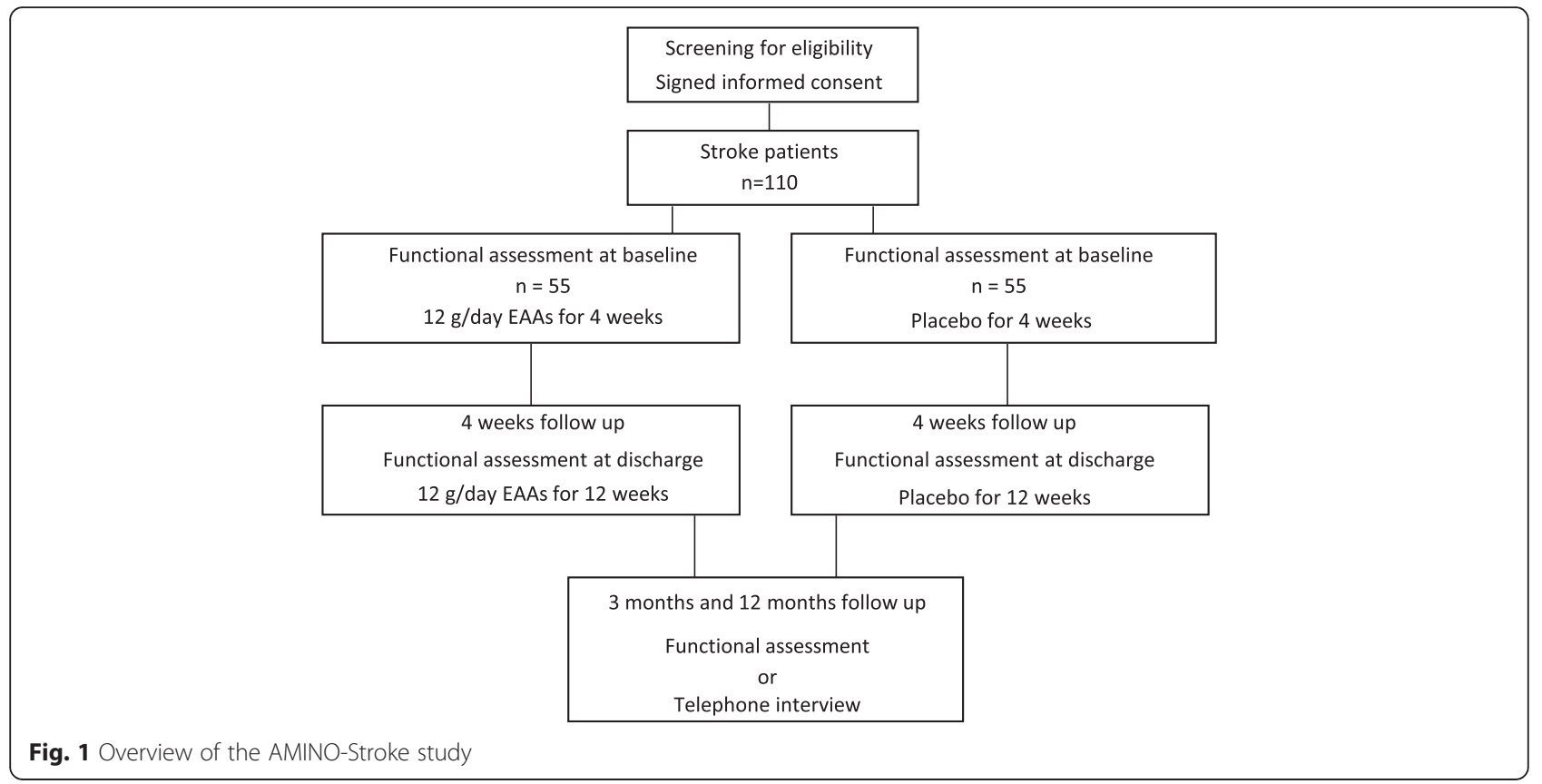

of motor function, balance, and muscle tone [20]. FuglMeyer scale is a system for the evaluation of motor function, balance, sensory qualities, and joint function in hemiplegic patients. This system applies a cumulative numerical score [21, 22]. Functional Ambulation Category $(F A C)$ includes six categories ranging from 0 to 5 and is defined by ability of the patient to ambulate, with a lowest score 0 for "patient cannot ambulate" to 5 "patient can ambulate independently" [23].

\section{Functional independency assessment}

Barthel index (BI) will be used for the assessment of functional independence. BI includes 10 basic aspects of activities related to self-care and mobility with scores of $0-100$, where the lower scores indicate greater dependency [24]. Short physical performance battery test (SPPBT) includes examination of ability to stand with the feet together in the side-by-side, semi-tandem, and tandem positions, time to walk 3 or 4 meters, and time to rise from the chair and return to the seated position 5 times [25].

\section{Nutritional status and quality of life}

Mini Nutritional Assessment (MNA) is a simple scale to measure nutritional status [26]. A score above 24 points identifies patients with a good nutritional status, whereas a score below 17 points reveals patients with malnutrition. Subjective Global Assessment (SGA) assesses nutritional status based on features of the history and physical examination [27]. EQ-5D questionnaire is an instrument for the generic measurements of health-related quality of life, comprising a Visual Analogue Scale for self-rating of general health and five domains evaluating mobility, self-care, usual activities, pain/discomfort, and anxiety/depression [28]. A score of 1 is the best possible general health status in each case; the dimensions may also be expressed as percentage of patients scoring from 1 to 3 (best to worst health status) or percentage of patients with 'any' complaint (scoring 2 or 3 in each domain) [29].

\section{Body composition analysis}

Body composition analysis at baseline and follow-up visits will be assessed by BIA device (BodyStat QuadScan 4000, Bodystat Limited, British Isles). The BIA device measures resistance $(R)$, reactance $(X c)$ and phase angle $(\phi)$. The measurements will be performed in patients in supine position lying on a non-conducting surface with arms slightly abducted from the trunk and legs slightly separated, after resting at least for $20 \mathrm{~min}$ [30]. Surface electrodes will be placed on the dorsal surface of hand and foot of the similar side. Skeletal muscle (SM) mass will be estimated according to the equation of Baumgartner at all [31].

\section{Serum biomarker assessment}

Blood samples and urine status will be assessed at admission and discharge from the inpatient rehabilitation as well as at follow up visits for routine clinical parameters, inflammatory and metabolic markers, and safety analyses (liver and kidney function). 


\section{Sample size calculation and statistical analyses}

A total of 110 patients is planned to be enrolled in the study. A prospective sample size calculation has been performed based on observational data from our study centre. A sample size of 55 in each group will have $80 \%$ power to detect a clinically relevant difference in Rivermead Motor Assessment Gross Function scale improvement between the groups of 1.5 points and a clinically relevant improvement of handgrip strength. The analysis of primary endpoints will use the principle of intentionto-treat. The carry-forward principle will be adopted for unavailable or missing data. Treatment effect will be assessed by unpaired comparison of both treatment arms for the change from baseline using the Student t-test. A significance level of $\alpha<0.05$ will be specified to indicate statistical significance. Secondary endpoints will be analyzed using standard statistical methods. For safety endpoints Chi-squared test of Fisher's Exact test will be used for event rates (e.g., death, re-stroke, hospitalization, cardiovascular event)

\section{Discussion}

The present AMINO Stroke study will be the first randomized double-blinded trial investigating an effect of essential amino acids (EAAs) on skeletal muscle functional capacity, muscle mass, muscle strength and physical performance in early post-stroke rehabilitation. In addition, the effect of EAAs supplementation on strokerelated quality of life, nutritional status, activities of daily life and outcome will be evaluated.

An improvement of physical and functional performance in stroke patients is expected after participating in 4-weeks rehabilitation program [32]. In the present study we expect a beneficial impact of the dietary supplementation of the EAA on skeletal muscle function and muscle mass during post-stroke rehabilitation.

The benefit of EAAs supplementation on skeletal muscle has been shown in several studies in elderly individuals. Thus, an elevated level of muscle protein synthesis was dependent on the availability of EAAs in a dose-depended manner [33]. An experimental study performing in the young adults revealed an increase of leucine incorporation into the skeletal muscle proteins under administration of EEAs as a flooding dose [34]. Ingestion of EAAs shortly after exercise enhanced muscle protein synthesis in older adults, indicating a combination of EAAs with exercising as a sufficient therapy for the treatment of muscle wasting [35]. Another study showed an improvement of lean mass, muscle functional capacity and muscle strength after 16 weeks supplementation of EAAs and arginine in healthy elderly individuals [36].

A reduction of the muscle mass might be directly depending on a limited availability of the EEAs and other nutrients [14]. Impaired feeding which results in malnutrition is frequently observed in patients with dysphagia after stroke [37]. About $50 \%$ of stroke patients have feeding difficulties due to dysphagia [38]. In older patients reduced nutritional status might be observed already at hospital admission with the diagnosis of acute stroke [39]. Patients with lower body weight have been observed to have worse fatal and non-fatal outcome after stroke [40]. While the inverse association of overweight and improved outcome has previously been termed obesity paradox [41], the perception advanced towards an obesity paradigm, acknowledging the protective overweight in cardiovascular disease [42].

Malnutrition and sarcopenia are often reported in the rehabilitation setting $[43,44]$. Therefore, we believe that supplementation of the EAAs will contribute to attenuation of muscle wasting and improve skeletal muscle recovery [45]. There are a number of methods available to assess muscle mass [46]. In the present study muscle mass will be estimated by using BIA that provides information to integrity of cell membrane and tissue interfaces, intracellular and extracellular fluid distribution, body fat and body lean mass [31, 47]. Previously, a strong correlation between muscle mass assessed by BIA and magnetic resonance imaging has been shown [48]. The prevalence of sarcopenia using BIA measurements has been repeatedly examined in elderly $[49,50]$, and in patients with neuromuscular or cerebrovascular disease $[51,52]$. Therefore we believe that BIA is an appropriate method for muscle mass assessment.

In Germany, stroke has not been selected for chronic disease management programs so far [53]. Post-stroke rehabilitation is usually recommended to start immediately following hospital discharge. The so called early post-stroke rehabilitation phase includes about 4 weeks. Mainly, recommendations given in discharge letters from acute care hospitals are frequently focusing on medical treatments [53]. At present, post-stroke management does not include monitoring of muscle mass, muscle strength and muscle function. The European stroke guidelines do not consider post-stroke muscle wasting as a relevant complication [54]. However, recognition of this problem through the medical community might improve the long-term outcome after stroke.

\footnotetext{
Abbreviations

EEA: Essential amino acid; BI: Barthel Index; EQ-5D: Quality of life questionnaire; RMA: Rivermead motor assessment; MAS: Motor assessment scale; FAC: Functional ambulation category; SPPBT: Short physical performance battery test; MNA: Mini nutritional assessment; SGA: Subjective global assessment; BIA: Bioelectrical impedance analysis; SM: Skeletal muscle.
}

\section{Competing interests}

KGH reports lecture fees and study grants by Bayer Healthcare, lecture fees and a study grant by Sanofi, lecture fees and advisory board fees from Pfizer, lecture fees from Bristol-Myers Squibb as well as advisory board fees from Edwards Lifesciences. Other authors report no competing interests. 


\section{Authors' contribution}

WD, UD and MJ intended this analysis and designed the study. WD performed the statistical calculations. NS wrote the manuscript. NS, NE, AS, $\mathrm{AM}, \mathrm{KGH}, \mathrm{SvH}, \mathrm{SDA}$ made substantial contributions to the study design. NE initiated the registry of the trial. All authors contributed relevant intellectua content to the manuscript and approved the final version.

\section{Acknowledgments}

We thank Mrs. Anja Kresse for the valuable assistance in the preparation and documentation of this study and Dr. Francesco S. Dioguardi for providing the EAAs supplementation.

\section{Funding}

The Center for Stroke Research Berlin (CSB) is an integrated research and treatment centre. The AMINO Stroke Study receives funding from German Federal Ministry of Education and Research (BMBF; grant number BMBF 01EO1301).

\section{Author details}

${ }^{1}$ Center for Stroke Research Berlin CSB, Charité - Universitätsmedizin Berlin, Berlin, Germany. ${ }^{2}$ German Centre for Cardiovascular Research (DZHK), partner site Berlin, Berlin, Germany. ${ }^{3}$ Innovative Clinical Trials, Department of Cardiology and Pneumology, University MedicineGöttingen (UMG), Göttingen, Germany. ${ }^{4}$ Department of Neurology, Charité - Universitätsmedizin Berlin, Berlin, Germany. ${ }^{5}$ Department of Neurology, Brandenburgklinik Bernau, Bernau, Germany. ${ }^{6}$ Department of Cardiology, Charité - Universitätsmedizin Berlin, Berlin, Germany.

\section{Received: 24 October 2015 Accepted: 14 January 2016}

\section{Published online: 22 January 2016}

\section{References}

1. Scherbakov N, Doehner W. Sarcopenia in stroke-facts and numbers on muscle loss accounting for disability after stroke. J Cachexia Sarcopenia Muscle. 2011;2:5-8.

2. Scherbakov N, Sandek A, Doehner W. Stroke-related sarcopenia: specific characteristics. J Am Med Dir Assoc. 2015;16:272-6.

3. Carin-Levy G, Greig C, Young A, Lewis S, Hannan J, Mead G. Longitudinal changes in muscle strength and mass after acute stroke. Cerebrovasc Dis. 2006;21:201-7.

4. Jørgensen $L$, Jacobsen BK. Changes in MM, fat mass, and bone mineral content in the legs after stroke: a 1 year prospective study. Bone. 2001;28: 655-9.

5. Rosenberg $\mathbb{H}$. Sarcopenia: origins and clinical relevance. J Nutr. 1997;127: 990S-1.

6. Boirie Y. Physiopathological mechanism of sarcopenia. J Nutr Health Aging 2009:13:717-23.

7. Scherbakov N, von Haehling S, Anker SD, Dirnagl U, Doehner W. Stroke induced Sarcopenia: muscle wasting and disability after stroke. Int J Cardiol. 2013;170:89-94.

8. Scherbakov N, Dirnagl U, Doehner W. Body weight after stroke: lessons from the obesity paradox. Stroke. 2011:42:3646-50.

9. Springer J, Schust S, Peske K, Tschirner A, Rex A, Engel O, et al. Catabolic signaling and muscle wasting after acute ischemic stroke in mice: indication for a stroke-specific sarcopenia. Stroke. 2014:45:3675-83.

10. Jørgensen HS, Nakayama H, Raaschou HO, Vive-Larsen J, Støier M, Olsen TS. Outcome and time course of recovery in stroke. Part II: Time course of recovery. The Copenhagen Stroke Study. Arch Phys Med Rehabil. 1995;76:406-12.

11. Bamford J, Sandercock P, Dennis M, Burn J, Warlow C. A prospective study of acute cerebrovascular disease in the community: the Oxfordshire Community Stroke Project-1981-86. 2. Incidence, case fatality rates and overall outcome at one year of cerebral infarction, primary intracerebral and subarachnoid haemorrhage. J Neurol Neurosurg Psychiatry. 1990;53:16-22.

12. Rondanelli M, Opizzi A, Antoniello N, Boschi F, ladarola P, Pasini E, et al. Effect of essential amino acid supplementation on quality of life, amino acid profile and strength in institutionalized elderly patients. Clin Nutr. 2011;30:571-7.

13. Bauer JM, Verlaan S, Bautmans I, Brandt K, Donini LM, Maggio M, et al Effects of a Vitamin D and Leucine-Enriched Whey Protein Nutritional Supplement on Measures of Sarcopenia in Older Adults, the PROVIDE Study: A Randomized, Double-Blind, Placebo-Controlled Trial. J Am Med Dir Assoc. 2015;16:740-7.
14. Dioguardi FS. Clinical use of amino acids as dietary supplement: pros and cons. J Cachexia Sarcopenia Muscle. 2011;2:75-80.

15. Volpi E, Kobayashi H, Sheffield-Moore M, Mittendorfer B, Wolfe RR. Essential amino acids are primarily responsible for the amino acid stimulation of muscle protein anabolism in healthy elderly adults. Am J Clin Nutr. 2003;78: 250-8.

16. Lincoln N, Leadbitter D. Assessment of motor function in stroke patients. Physiotherapy. 1979;65:48-51.

17. Collen FM, Wade DT, Robb GF, Bradshaw CM. The Rivermead Mobility Index: a further development of the Rivermead Motor Assessment. Int Disabil Stud. 1991;13:50-4.

18. Bohannon RW. Adequacy of hand-grip dynamometer for characterizing upper limb strength after stroke. Isokinet Exerc Sci. 2004;12:263-5.

19. Mathiowetz V, Weber K, Volland G, Kashman N. Reliability and validity of grip and pinch strength evaluations. J Hand Surg [Am]. 1984;9:222-6.

20. Carr JH, Shepherd RB, Nordholm L, Lynne D. Investigation of a new motor assessment scale for stroke patients. Phys Ther. 1985;65:175-80.

21. Fugl-Meyer AR, Jääskö L, Leyman I, Olsson S, Steglind S. The post-stroke hemiplegic patient. 1. a method for evaluation of physical performance. Scand J Rehabil Med. 1975;7:13-31.

22. Sullivan KJ, Tilson JK, Cen SY, Rose DK, Hershberg J, Correa A, et al. FuglMeyer assessment of sensorimotor function after stroke: standardized training procedure for clinical practice and clinical trials. Stroke. 2011;42: 427-32.

23. Holden MK, Gill KM, Magliozzi MR, Nathan J, Piehl-Baker L. Clinical gait assessment in the neurologically impaired. Reliability and meaningfulness. Phys Ther. 1984;64:35-40.

24. Kasner SE. Clinical interpretation and use of stroke scales. Lancet Neurol. 2006:5:603-12.

25. Guralnik JM, Simonsick EM, Ferrucci L, Glynn RJ, Berkman LF, Blazer DG, et al. A short physical performance battery assessing lower extremity function: association with self-reported disability and prediction of mortality and nursing home admission. J Gerontol. 1994;49:M85-94.

26. Vellas B, Villars H, Abellan G, Soto ME, Rolland Y, Guigoz Y, et al. Overview of the MNA-Its history and challenges. J Nutr Health Aging. 2006;10:456-65.

27. Detsky AS, McLaughlin JR, Baker JP, Johnston N, Whittaker S, Mendelson RA, et al. What is subjective global assessment of nutritional status? JPEN J Parenter Enteral Nutr. 1987:11:8-13.

28. Devlin NJ, Krabbe PF. The development of new research methods for the valuation of EQ-5D-5 L. Eur J Health Econ. 2013;14:S1-3.

29. Comin-Colet J, Lainscak M, Dickstein K, Filippatos GS, Johnson P, Lüscher TF, et al. The effect of intravenous ferric carboxymaltose on health-related quality of life in patients with chronic heart failure and iron deficiency: a subanalysis of the FAIR-HF study. Eur Heart J. 2013;34:30-8.

30. Lukaski HC, Johnson PE, Bolonchuk WW, Lykken Gl. Assessment of fat-free mass using bioelectrical impedance measurements of the human body. Am J Clin Nutr. 1985:41:810-7.

31. Baumgartner RN, Chumlea WC, Roche AF. Bioelectric impedance phase angle and body composition. Am J Clin Nutr. 1988;48:16-23.

32. Scherbakov N, Knops M, Ebner N, Valentova M, Sandek A, Grittner U, et al. Evaluation of C-terminal Agrin Fragment as a marker ofmuscle wasting in patients after acute stroke during early rehabilitation. J Cachexia Sarcopenia Muscle. doi:10.1002/jcsm.12068

33. Cuthbertson D, Smith K, Babraj J, Leese G, Waddell T, Atherton P, et al. Anabolic signaling deficits underlie amino acid resistance of wasting, aging muscle. FASEB J. 2005;19:422-4.

34. Smith K, Reynolds N, Downie S, Patel A, Rennie MJ. Effects of flooding amino acids on incorporation of labeled amino acids into human muscle protein. Am J Physiol. 1998;275:E73-8.

35. Walker DK, Dickinson JM, Timmerman KL, Drummond MJ, Reidy PT, Fry CS, et al. Exercise, amino acids, and aging in the control of human muscle protein synthesis. Med Sci Sports Exerc. 2011:43:2249-58.

36. Børsheim E, Bui QU, Tissier S, Kobayashi H, Ferrando AA, Wolfe RR. Effect of amino acid supplementation on MM, strength and physical function in elderly. Clin Nutr. 2008;27:189-95

37. Foley NC, Martin RE, Salter KL, Teasell RW. A review of the relationship between dysphagia and malnutrition following stroke. J Rehabil Med. 2009;41: 707-13.

38. Geeganage C, Beavan J, Ellender S, Bath PM. Interventions for dysphagia and nutritional support in acute and subacute stroke. Cochrane Database Syst Rev. 2012;10:CD000323. 
39. Pirlich M, Schütz T, Norman K, Gastell S, Lübke HJ, Bischoff SC, et al. The German hospital malnutrition study. Clin Nutr. 2006;25:563-72.

40. Doehner W, Schenkel J, Anker SD, Springer J, Audebert HJ. Overweight and obesity are associated with improved survival, functional outcome, and stroke recurrence after acute stroke or transient ischaemic attack: observations from the TEMPiS trial. Eur Heart J. 2013;34:268-77.

41. Lainscak M, von Haehling S, Doehner W, Anker SD. The obesity paradox in chronic disease: facts and numbers. J Cachexia Sarcopenia Muscle. 2012;3:1-4.

42. Doehner W, von Haehling S, Anker SD. Protective overweight in cardiovascular disease: moving from 'paradox' to 'paradigm'. Eur Heart J. 2015. (pubmed ahead of print).

43. Wakabayashi H, Sakuma K. Rehabilitation nutrition for sarcopenia with disability: a combination of both rehabilitation and nutrition care management. J Cachexia Sarcopenia Muscle. 2014:5:269-77.

44. Michel JP. Sarcopenia: there is a need for some steps forward. J Am Med Dir Assoc. 2014;15:379-80.

45. Malafarina V, Uriz-Otano F, Iniesta R, Gil-Guerrero L. Effectiveness of nutritional supplementation on muscle mass in treatment of sarcopenia in old age: a systematic review. J Am Med Dir Assoc. 2013;14:10-7.

46. Heymsfield SB, Adamek M, Gonzalez MC, Jia G, Thomas DM. Assessing skeletal muscle mass: historical overview and state of the art. J Cachexia Sarcopenia Muscle. 2014;5:9-18

47. Norman K, Wirth R, Neubauer M, Eckardt R, Stobäus N. The bioimpedance phase angle predicts low muscle strength, impaired quality of life, and increased mortality in old patients with cancer. J Am Med Dir Assoc. 2015; 16:173. e17-22

48. Janssen I, Heymsfield SB, Baumgartner RN, Ross R. Estimation of skeletal MM by bioelectrical impedance analysis. J Appl Physiol (1985). 2000;89:465-71.

49. Janssen I, Heymsfield SB, Ross R. Low relative skeletal MM (sarcopenia) in older persons is associated with functional impairment and physical disability. J Am Geriatr Soc. 2002;50:889-96.

50. Legrand D, Vaes B, Matheï C, Swine C, Degryse JM. The prevalence of sarcopenia in very old individuals according to the European consensus definition: insights from the BELFRAlL study. Age Ageing. 2013:42:727-34.

51. Rutkove SB, Aaron R, Shiffman CA. Localized bioimpedance analysis in the evaluation of neuromuscular disease. Muscle Nerve. 2002:25:390-7.

52. Sotillo C, López-Jurado M, Martín E, Mataix J, Llopis J. Body composition assessed by anthropometry and bioelectric impedance analysis in older persons recovering from cerebrovascular accident. Int J Vitam Nutr Res. 2003;73:32-8.

53. Leistner S, Michelson G, Laumeier I, Ahmadi M, Smyth M, Nieweler G, et al. Intensified secondary prevention intending a reduction of recurrent events in TIA and minor stroke patients (INSPiRE-TMS): a protocol for a randomised controlled trial. BMC Neurol. 2013;13:11.

54. European Stroke Organisation (ESO) Executive Committee, ESO Writing Committee. Guidelines for management of ischaemic stroke and transient ischaemic attack 2008. Cerebrovasc Dis. 2008;25(5):457-507.

\section{Submit your next manuscript to BioMed Central and we will help you at every step:}

- We accept pre-submission inquiries

- Our selector tool helps you to find the most relevant journal

- We provide round the clock customer support

- Convenient online submission

- Thorough peer review

- Inclusion in PubMed and all major indexing services

- Maximum visibility for your research

Submit your manuscript at www.biomedcentral.com/submit

) Biomed Central 